УДК 517.55

\title{
On Domains of Convergence of Multidimensional Lacunary Series
}

\author{
Taxir T. Tuychiev* \\ National University of Uzbekistan \\ VUZ Gorodok, Tashkent, 100174 \\ Uzbekistan
}

Received 06.04.2019, received in revised form 06.07.2019, accepted 06.08.2019

This paper is devoted to multidimensional analogues of the Fabry and Polya theorems on lacunary series. Domains of convergence of lacunary Hartogs series and series in homogeneous polynomials are studied in this paper. Analogues of the Fabry and Polya theorems for such series are given and domains of convergence of these series are described. Results of the work develop well-known result of J. Siciak on the domain of convergence of the lacunary series with respect to homogeneous polynomials.

Keywords: plurisubharmonic function, singular point, negligible sets in $\mathbb{C}^{n}$, power series, lacunar Hartogs series, series in homogeneous polynomials.

DOI: 10.17516/1997-1397-2019-12-6-736-746.

\section{Introduction}

This paper is devoted to multidimensional analogues of the Fabry and Pólya theorems on lacunary series. We study domains of convergence of lacunary Hartogs series and series in homogeneous polynomials. Analogues of the Fabry and Pólya theorems for such series are given.

We consider the following lacunary series on the plane

$$
f(z)=\sum_{k=0}^{\infty} a_{n_{k}} z^{n_{k}}, \varlimsup_{k \rightarrow \infty}\left|a_{n_{k}}\right|^{\frac{1}{n_{k}}}=1 .
$$

Then it is clear that the radius of convergence of this series is 1 and this series converges inside the unit disk $U=\{z:|z|<1\}$. There is at least one singular point of function $f$ on the boundary $\partial U$.

The case when each boundary point of the unit disk is singular point of $f(z)$ is interesting, that is, the unit disk $U$ is the natural Weierstrass domain for the function $f(z)$.

It is well known that each boundary point of the disk of convergence for the series

$$
f(z)=\sum_{k=0}^{\infty} z^{2^{k}}
$$

is singular. Therefore the disk of convergence $U=\{z:|z|<1\}$ is the natural Weierstrass domain for the function $f(z)$.

The answer to the question of when each boundary point of the domain of convergence is a singular point for the sum of a series is in the following statement (the Fabry theorem [1]): if for series (1)

$$
\varlimsup_{k \rightarrow \infty} \frac{k}{n_{k}}=0
$$

tahir1955@mail.ru

(c) Siberian Federal University. All rights reserved 
then the circle $|z|=1$ is natural the boundary of $f(z)$, i.e., $f(z)$ is single-valued holomorphic function in $\{|z|<1\}$, and each point of the circle $|z|=1$ is a singular point of function $f(z)$, $W_{f}(z)=\{|z|<1\}$.

Faber constructed an example of a series (see [2]) for which

$$
\varliminf_{k \rightarrow \infty} \frac{k}{n_{k}}=0
$$

and the sum of the series has only one singular point on $|z|=1$.

Nevertheless, under condition (3) the following statement holds (the Polya theorem [3]): if condition (3) holds for series (1) then series (1) defines a single-valued holomorphic function with a simply-connected domain of existence $W_{f} \subset \mathbb{C}$.

It is known that the Hartogs series and series in homogeneous polynomials play an important role in multidimensional complex analysis. In this paper we study domains of convergence of such series.

In Section 1 of this paper we describe the domains of convergence of series in homogeneous polynomials that converge on a pencil of complex lines in terms of the Green's function. In Section 2 we study the domain of convergence of the Hartogs series, assuming only that coefficients are holomorphic.

In Sections 3 and 4 we give multidimensional analogues of the Fabry and Pólya theorems for lacunary Hartogs series and series in homogeneous polynomials. In particular, in section 5 we consider the Siciak's theorem [4], which is a multidimensional analogue of the Fabry theorem on gaps for series in homogeneous polynomials. We give another proof of this theorem based on pluripotential theory.

This work was initiated by the above-mentioned article of J.Siciak and his discussion with A. Sadullaev on the possible applications of pluripotential theory to proofs of multidimensional analogues of the Fabry and Pólya theorems. I would like to express my sincere gratitude to professor J. Siciak for formulating problems and to professor A. Sadullaev for useful discussions and assistance in the work.

\section{The domain of convergence of series of homogeneous polynomials with variable radius of convergence}

In this section we study the question of the holomorphic continuation of series in homogeneous polynomials

$$
\sum_{s=0}^{\infty} \mathbb{Q}_{s}(z),
$$

assuming only that for each complex line $\ell \ni 0$ in some family $\mathcal{L} \subset \mathbb{P}^{n-1}$ this series converges in a disk $\ell \cap B(0, r(\ell))$ of radius $r(\ell)>0$.

Further we shall use the following definitions and facts from the multidimensional complex potential theory [5]- [7].

Definition 1. A subset $E$ of a domain $D \subset \mathbb{C}^{n}$ is said to be pluripolar in a domain $D$ if there exists a function $u(z)$ that is plurisubharmonic in $D$ such that $u \not \equiv-\infty$, but $\left.u\right|_{E}=-\infty$

We denote the class of functions $u(z)$ that are plurisubharmonic in $\mathbb{C}^{n}$ by $\mathrm{L}$ and such that $u(z) \leqslant \beta_{u}+\ln (1+|z|)$ for all $z \in \mathbb{C}^{n}$.

Definition 2. Let $E \subset \mathbb{C}^{n}$ be an arbitrary set and $L(E)$ be a class of functions $u \in L$ such that $\left.u\right|_{E} \leqslant 0$. We put

$$
V(z, E)=\sup \{u(z): u \in L(E)\}
$$


Then function

$$
V^{*}(z, E)=\varlimsup_{w \rightarrow z} V(w, E)
$$

is called Green's function of the set $E$ (or the Siciak-Zakharyuta extremal function).

Note that if the set $E \subset \mathbb{C}^{n}$ is pluripolar in $\mathbb{C}^{n}$ then $V^{*}(z, E) \equiv+\infty$ and vice versa. If $V^{*}(z, E) \equiv+\infty$ then there exists a plurisubharmonic function $u(z) \in L, u \not \equiv-\infty$ such that $\left.u\right|_{E}=-\infty$.

It is well known that any function $f(z)$ that is holomorphic in a complete circular domain $D \subset \mathbb{C}^{n}$ and centered at point $z=0$ in this domain can be expanded in a series in homogeneous polynomials

$$
f(z)=\sum_{s=0}^{\infty} \mathbb{Q}_{s}(z) .
$$

The series converges uniformly on compact subsets of $D$.

Note that not every complete circular domain is a domain of convergence of a series in homogeneous polynomials. The situation here is the same as in the case of the Hartogs or Reinhart series. If we take the transformation

$$
\left({ }^{\prime} z, z_{n}\right) \rightarrow\left({ }^{\prime} w, z_{n}\right), \text { where } w_{\nu}=\frac{z_{\nu}}{z_{n}}(\nu=1,2, \ldots, n-1)
$$

that transforms the circular domain into the Hartogs domain then series (5) becomes

$$
g\left({ }^{\prime} w, z_{n}\right)=f\left({ }^{\prime} w z_{n}, z_{n}\right)=\sum_{s=0}^{\infty} Q_{s}\left({ }^{\prime} w, 1\right) z_{n}^{s}
$$

Thus we obtain a Hartogs series.

To find domains of convergence of series in homogeneous polynomials we need the following statement that relates the extremal Green's function to a polynomial (see [5]).

Proposition 1. For any compact $K \subset \mathbb{C}^{n}$

$$
V^{*}(z, K)=\left\{\sup \left(\frac{1}{\operatorname{deg} P} \ln |P(z)|:\|P\|_{K} \leqslant 1\right)\right\}^{*} .
$$

Using (6), we prove the following statement for series in homogeneous polynomials with variable radius of convergence.

Theorem 1. Let us assume that a formal series in homogeneous polynomials $\mathbb{Q}_{s}$

$$
\sum_{s=0}^{\infty} \mathbb{Q}_{s}(z)
$$

and the family $\mathcal{L} \subset \mathbb{P}^{n-1}$ of complex lines $\ell$ are given. If for every complex line $\ell \in \mathcal{L}$ the series $\left.\sum_{s=0}^{\infty} \mathbb{Q}_{s}(z)\right|_{l}$ converges in the disk $\ell \cap B(0, r(l)), 0<r(l) \leqslant 1$ then it converges uniformly inside the domain

$$
\Omega=\left\{z \in \mathbb{C}^{n}:|z| \cdot \exp V^{*}\left(\frac{z}{|z|}, E\right)<1\right\}
$$

where $E=\bigcup_{l \in L}(l \cap B(0, r(l)))$.

Since the generalized Green's function $V^{*}(z, E) \equiv+\infty$ if and only if $E$ is pluripolar set in $\mathbb{C}^{n}$ then it follows from (7) that Theorem 1 is meaningful only if the set is non-pluripolar as we will assume.

Proof of Theorem 1. Let $0<r<1$ be an arbitrary number and 


$$
E_{r}=\left\{\bigcup_{l \in L}(l \cap B(0, r(l)): r(l) \geqslant r\} .\right.
$$

Then it is clear that $E_{r_{1}} \subset E_{r_{2}}$ on $r_{1}>r_{2}$ and $\bigcup_{r=0}^{1} E_{r}=E$. We fix the number $r>0$ so that the set $E_{r}$ is non-pluripolar (this is possible because of nonpluripolarity of $E$ ). Consequently, by Proposition $1[6]$ the series converges uniformly in some neighbourhood of zero and the sum of this series determines some function $f(z)$ :

$$
f(z):=\sum_{s=0}^{\infty} \mathbb{Q}_{s}(z) .
$$

From this and conditions of the theorem it follows that function $f(z)$ satisfies the following conditions:

1) $f(z) \in \mathcal{O}\{0\}$,

2) for each complex line $l \in \mathcal{L}$ the function $f$ is holomorphic in the disk $l \cap B(0, r(l))$, that is,

$$
\left.f\right|_{l \in \mathcal{L}} \in \mathcal{O}\{l \cap B(0, r(l))\} .
$$

Let us fix now an arbitrary small number $\varepsilon, 0<\varepsilon<r$, and put

$$
\mathcal{L}_{r, m}=\left\{l \in \mathcal{L}: r(l) \geqslant r,|f|_{l \cap B(0, r(l)-\varepsilon)} \leqslant m\right\}, m=1,2, \ldots .
$$

Let $l \in \mathcal{L}_{r, m}, z \in l$, and $\omega=\frac{r(l) z}{|z|}$. Then the complex line

$$
l=\{\xi: \xi=\omega \lambda, \lambda \in \mathbb{C}\}
$$

passes through a point $z$. We take the restriction of the function $f$ to this line

$$
\varphi(\lambda)=f(\lambda \omega)=\sum_{s=0}^{\infty} \mathbb{Q}_{s}(\lambda \omega)=\sum_{s=0}^{\infty} \mathbb{Q}_{s}(\omega) \lambda^{s} .
$$

This series converges in a disk $|\lambda|<1$. According to (8)

$$
|\varphi(\lambda)|=|f(\lambda \omega)| \leqslant m \text { at }|\lambda \omega| \leqslant r(l)-\varepsilon,
$$

i.e., $|\lambda| \leqslant \frac{r(l)-\varepsilon}{|\omega|}=\frac{r(l)-\varepsilon}{r(l)}=1-\frac{\varepsilon}{r(l)} \leqslant 1-\delta$, where $\delta=\frac{\varepsilon}{r}, 0<\delta<1$.

Hence, according to the Cauchy inequalities

$$
\left|\mathbb{Q}_{s}(\omega)\right| \leqslant \frac{m}{(1-\delta)^{s}}, \quad s=0,1,2, \ldots
$$

These inequalities are true for all $\omega \in \bigcup_{l \in \mathcal{L}_{r, m}}(l \cap B(0, r(l)-\varepsilon))=E_{r, m}$. By the Bernstein-Walsh inequality (see (6)) we have

$$
\left|\mathbb{Q}_{s}(z)\right| \leqslant \frac{m}{(1-\delta)^{s}} \cdot\left[\exp V^{*}\left(z, E_{r, m}\right)\right]^{s}, \quad z \in \mathbb{C}^{n} .
$$

Therefore, using equality

$$
\mathbb{Q}_{s}(z)=|z|^{s} \mathbb{Q}_{s}\left(\frac{z}{|z|}\right)
$$


and inequality (9) we obtain the following inequality

$$
\left|\mathbb{Q}_{s}(z)\right| \leqslant \frac{|z|^{s} \cdot m}{(1-\delta)^{s}} \cdot\left[\exp V^{*}\left(\frac{z}{|z|}, E_{r, m}\right)\right]^{s}, \quad z \in \mathbb{C}^{n}
$$

It follows that series $\sum_{s=0}^{\infty} \mathbb{Q}_{s}(z)$ converges uniformly inside the set

$$
\Omega_{r, m}=\left\{z \in \mathbb{C}^{n}: \frac{|z|}{1-\delta} \cdot \exp V^{*}\left(\frac{z}{|z|}, E_{r, m}\right)<1\right\}
$$

(Recall that the domain of convergence of a series $\sum_{s=0}^{\infty} \mathbb{Q}_{s}(z)$, where $\mathbb{Q}_{s}(z)$ is a homogeneous polynomial of degree $s$, is a set $\left.\left\{z \in \mathbb{C}^{n}: \varlimsup_{S \rightarrow \infty}\left|\mathbb{Q}_{s}(z)\right|^{\frac{1}{s}}<1\right\}\right)$.

Now we take $\varepsilon \rightarrow o$ and $m \rightarrow \infty$ in (10). Then we obtain the convergence of series (4) inside the domain

$$
\Omega_{r}=\left\{z \in \mathbb{C}^{n}:|z| \cdot \exp V^{*}\left(\frac{z}{|z|}, E_{r}\right)<1\right\}
$$

From this $r \searrow 0$ we obtain that series $\sum_{s=0}^{\infty} \mathbb{Q}_{s}(z)$ converges in the domain

$$
\Omega=\left\{z \in \mathbb{C}^{n}:|z| \cdot \exp V^{*}\left(\frac{z}{|z|}, E\right)<1\right\}
$$

The theorem is proved.

We remark that if we set $r(\ell) \equiv 1$ in Theorem 1 then we obtain the well-known result of A. Sadullaev (Proposition 1, [5]). If we set $r(\ell) \equiv 1$ and $E=B(0,1)$ then we obtain the Forelli theorem. Moreover, if the radius of convergence $r(\ell) \leqslant R$ for all $l \in E$ then it is not difficult to prove that the domain of convergence $\Omega$ in (7) can be replaced by $\Omega_{R}=$ $=\left\{z \in \mathbb{C}^{n}: \frac{|z|}{R} \cdot \exp V^{*}\left(\frac{R z}{|z|}, E\right)<1\right\}$. It is clear that as $\mathrm{R}$ is increased the domain is also increased. Therefore, series (4) actually converges inside

$$
\Omega=\bigcup_{R} \Omega_{R}=\lim _{R \rightarrow+\infty}\left\{z \in \mathbb{C}^{n}: \frac{|z|}{R} \cdot \exp V^{*}\left(\frac{R z}{|z|}, E\right)<1\right\}
$$

where the union is over all such $R$.

\section{Holomorphy of the sum of Hartogs series}

In this section we consider the holomorphy of the Hartogs series

$$
f(z, w)=\sum_{k=0}^{\infty} c_{k}(z) w^{k}
$$

assuming only that coefficients $c_{k}(z)$ are holomorphic. In this case we require the natural condition that radius of convergence $R(z)$ of series (11) is positive for each fixed point $z \in D$, where $D \subset \mathbb{C}_{z}^{n}$ is some domain. We note that if $f(z, w)$ is holomorphic function in a domain $D \times\{|w|<r\} \subset \mathbb{C}_{z}^{n} \times \mathbb{C}_{w}$ then $f(z, w)$ is represented in this domain by series (11), where $c_{k}(z)$ are holomorphic functions and the series converges uniformly inside $D \times\{|w|<r\}$. In our case, there is no convergence of series (11) in such domain. A similar problem was considered by M. Jarnicki, P. Pflug [8]. 
Theorem 2 (9). For arbitrary series (11) with coefficients $c_{k}(z)$ that are holomorphic in the domain $D \subset \mathbb{C}_{z}^{n}$ there exists a nowhere dense closed set $S \subset D$ such that

1) $-\ln R_{*}(z) \in \operatorname{psh}(D \backslash S)$,

2) the sum of series (11) is holomorphic for the set of variables in

$$
\left\{z \in(D \backslash S),|w|<R_{*}(z)\right\},
$$

where $R_{*}(z)=\varliminf_{w \rightarrow z} R(w)$ is the lower regularization of the radius of function $R(z)$.

Proof. Let us consider sets

$$
E_{m}=\left\{z \in D:\left|c_{k}(z)\right|^{\frac{1}{k}} \leqslant m, k=1,2, \ldots\right\}, \quad m=1,2, \ldots
$$

By construction and from the holomorphy of coefficients $c_{k}(z)$ in $D$ it follows that these sets are closed, $E_{1} \subset E_{2} \subset \ldots$ and $D=\bigcup_{m=1}^{\infty} E_{m}$. Then, by the Baire Theorem [10] there exists a set $E_{M}$ with non-empty interior $E_{M}^{0}$. By hypothesis, $\frac{1}{k} \ln \left|c_{k}(z)\right| \leqslant \ln M$ for all $z \in E_{M}^{0}, k=1,2, \ldots$.

Symbol $D_{1}$ denotes the set of points $z^{0} \in D$ such that in some neighbourhood $V \ni z^{0}$ all functions $\frac{1}{k} \ln \left|c_{k}(z)\right|$ are bounded from above by some constant $M\left(z^{0}\right)$, that is, $\frac{1}{k} \ln \left|c_{k}(z)\right| \leqslant$ $M\left(z^{o}\right)$ for any $z \in V$ and $k=1,2, \ldots$. Then the set $S=\bar{D} \backslash \stackrel{0}{D_{1}}$ is closed and nowhere dense in $D$. Indeed, if $S$ is not nowhere dense in $D$ then $S$ contains some neighbourhood $U \subset S$. Using results proved above, there exists a ball $B \subset S$ in which $\frac{1}{k} \ln \left|c_{k}(z)\right|$ are uniformly bounded from above, i.e. $\frac{1}{k} \ln \left|c_{k}(z)\right| \leqslant M$ for all $z \in B$ and $k=1,2, \ldots$. The contradiction proves that set $S$ is nowhere dense.

Now let

$$
R(z)=\frac{1}{\varlimsup_{k \rightarrow \infty}\left|c_{k}(z)\right|^{\frac{1}{k}}}
$$

be a radius of convergence of series (11). Then $-\ln R(z)=\varlimsup_{k \rightarrow \infty} \frac{1}{k} \ln \left|c_{k}(z)\right|$. Taking into account the plurisubharmonicity and local boundedness of functions $\frac{1}{k} \ln \left|c_{k}(z)\right|$ in $D_{1}$, we obtain that function $-\ln R_{*}(z)$ is plurisubharmonic in $D_{1}$, where $R_{*}(z)=\underline{\lim _{w \rightarrow z}} R(w)$ is the lower regularization of function $R(z)$. In addition, outside some pluripolar set $\Pi \subset D$ the equality $R_{*}(z)=R(z)$ holds (see [5]).

The holomorphy of the sum of series (11) in $\left\{z \in(D \backslash S),|w|<R_{*}(z)\right\}$ follows from the uniform convergence of series (11) inside this region. The theorem is proved.

Remark 1. The existence of $S$ in Theorem 2 is necessary. For a nowhere dense connected closed set $S \subset \mathbb{C}$ with a connected complement with respect to an infinitely distant point (that is, any point $z \in \mathbb{C} \backslash S$ can be joined by a continuous curve with an infinite point) we can construct a series $\sum_{k=0}^{\infty} c_{k}(z) w^{k}$ for which the set $S \times \mathbb{C}$ is an unremovable singular set ([8]).

\section{Multidimensional analogues of the Fabry and Pólya theorems for Hartogs series}

This section is devoted to multidimensional analogues of the Fabry and Pólya theorems on lacunary series. Here we study the domain of convergence of the lacunary Hartogs series and give analogues of the Fabry and Pólya theorems for such series. 
Let

$$
f(z, w)=\sum_{k=0}^{\infty} c_{n_{k}}(z) w^{n_{k}}
$$

be a lacunary Hartogs series with holomorphic coefficients $c_{n_{k}}(z) \in \mathcal{O}(D), k=0,1,2, \ldots$, where $D \subset \mathbb{C}^{n}$ is some region. In this case we require the natural condition that radius of convergence of series (12) $R(z)$ is positive for each fixed point $z \in D$.

Theorem 3. Let series (12) satisfies the following conditions

1) $c_{n_{k}}(z) \in \mathcal{O}(D), k=0,1,2, \ldots$, and for each fixed $z \in D$ it converges in the disk $|w|<$ $R(z), R(z)>0$,

2) $\varlimsup_{k \rightarrow \infty} \frac{k}{n_{k}}=0$.

Then there exist a closed nowhere dense set $S \subset D$ such that the sum of series $(12) f(z, w)$ is holomorphic for the set of variables inside the domain

$$
\left\{z \in D \backslash S,|w|<R_{*}(z)\right\}
$$

and each point of the set $\left\{z \in D \backslash S,|w|=R_{*}(z)\right\}$ is a singular point of function $f(z, w)$, where $R_{*}(z)=\varliminf_{w \rightarrow z} R(w)$ is the lower regularization of the radius of function $R(z)$.

Proof. By Theorem 2 there is nowhere dense closed set $S \subset D$ such that function $f(z, w)$ is holomorphic in the set of variables in

$$
\left\{z \in D \backslash S,|w|<R_{*}(z)\right\},
$$

where $R_{*}(z)=\varliminf_{w \rightarrow z} R(w)$ is the lower regularization of the radius of function $R(z)$. In addition, outside some pluripolar set $\Pi \subset D$ the equality $R_{*}(z)=R(z)$ holds (see [5]).

Using condition 2 of Theorem 3 and the classical Fabry theorem on gaps, we obtain that each point of the set $\left\{z \in D \backslash S,|w|==R_{*}(z)\right\}$ is a singular point of function $f(z, w)$. Indeed, in any neighbourhood of a point of this set there are infinitely many points that are singular points of $f(z, w)$. Consequently, the point itself is singular for this function. The theorem is proved. (12).

Now we consider the situation when condition (3) is satisfied for coefficients of lacunary series

In this case, we have the following multidimensional analogue of the Pölya theorem.

Theorem 4 ([11]). Let us assume that series (12) satisfies the following conditions:

1) series (12) converges uniformly inside the domain $D \times\{|w|<r\} \subset \mathbb{C}_{z}^{n} \times \mathbb{C}_{w}, r>0$;

2) $\lim _{k \rightarrow \infty} \frac{k}{n_{k}}=0$.

Then series (12) defines a single-valued holomorphic function $f(z, w)$ in the domain $W^{0}$, where $W^{0}$ is the open kernel of the set $W=\bigcup_{z \in D} W_{f}(z)$.

To prove this theorem we need the following statement.

Proposition 2. [12]. We consider function $f(z, w)$ that is holomorphic in the domain $D \times$ $\{|w|<r\} \subset \mathbb{C}_{z}^{n} \times \mathbb{C}_{w}, r>0$, such that for each fixed $z \in D$ function $f(z, w)$, the function holomorphic in the disk $|w|<r$ is single-valued in $\mathbb{C}_{w}$, that is, $W_{f}(z) \subset \mathbb{C}_{w}$.

We set $W=\bigcup_{z \in D} W_{f}(z)$. Then 
1) an open kernel $W^{0}$ is domain (that is, it is connected), and the set $P=n p_{D}\left(W / W^{0}\right)$ is pluripolar in $D$;

2) function $f(z, w)$ extends holomorphically to $W^{0}$;

3) if $\rho(z, w)=\rho\left(z, \partial W_{f}(z)\right)$ the distance from the point $(z, w)$ to the boundary $\partial W_{f}(z)$ is fixed $z \in D$,

then the regularization $(-\ln \rho(z, w))^{*}$ is a plurisubharmonic function in $W^{0}$.

Let us note that this proposition is connected with the well-known classical fact. Let function $f(z, w)$ be holomorphic in some domain $W^{0} \subset \mathbb{C}^{n+1}$ and not holomorphic to boundary points $\partial W^{0}$ and let $\rho(z, w)$ be the distance from the point $(z, w) \in W^{0}$ to the boundary $\partial W^{0}(z)=\partial W^{0} \cap\{z\}$, that is, Hartogs radius of the domain $W^{0}$ at the point $(z, w)$ belongs to $W^{0}$. Then function $-\ln \rho(z, w)$ is plurisubharmonic in $W^{0}$ [13]. The proposition does not require holomorphy $f$ in the set $W^{0}$ in summation of variables; it is proved under weak restrictions on $f$.

Proof of Theorem 4. It follows from condition 1) of Theorem 4 that $f(z, w) \in$ $\mathcal{O}(D \times\{|w|<r\}) \subset \mathbb{C}_{z}^{n} \times \mathbb{C}_{w}, r>0$. Using condition 2) and the classical Polya theorem, we obtain that for each fixed $z \in D$ function $f(z, w)$ holomorphic in the disk $|w|<r$ uniquely extends to $\mathbb{C}_{w}$, that is, $W_{f}(z) \subset \mathbb{C}_{w}$.

Then, by Proposition 2 the open kernel $W^{0}$ of the set $W=\bigcup_{z \in D} W_{f}(z)$ is a domain and function $f(z, w)$ extends holomorphically to $W^{0}$.

Using Theorems 2 and 4, we obtain the following multidimensional analogue of the Pólya theorem for the lacunary Hartogs series with variable radius of convergence.

Theorem 5. Assume that series (12) satisfies the following conditions:

1) $c_{n_{k}}(z) \in \mathcal{O}(D), k=0,1,2, \ldots$;

2) it converges for every fixed $z \in D$ in the disk $|w|<R(z), R(z)>0$;

3) $\varliminf_{k \rightarrow \infty} \frac{k}{n_{k}}=0$.

Then there exists a closed nowhere dense set $S \subset D$ such that series (12) defines a singlevalued holomorphic function $f(z, w)$ in the domain $W^{0} \backslash(S \times \mathbb{C})$ where $W^{0}$ is the open kernel of the set $W=\bigcup_{z \in D} W_{f}(z)$.

\section{Analogues of the Fabry and Pólya theorems for series in homogeneous polynomials. The Siciak's theorem}

Let us consider the formal lacunary series

$$
\sum_{k=0}^{\infty} \mathbb{Q}_{n_{k}}(z)
$$

in homogeneous polynomials $\mathbb{Q}_{n_{k}}(z)$ and we will study the domain of convergence of such series.

J. Siciak [4] considered the following situation. Let function $f(z)$ of $n$ complex variables be holomorphic in a neighbourhood of zero and it is represented in the form of a lacunary series in homogeneous polynomials

$$
f(z)=\sum_{k=0}^{\infty} \mathbb{Q}_{n_{k}}(z) .
$$


Let $\psi(z):=\varlimsup_{k \rightarrow \infty}\left|\mathbb{Q}_{n_{k}}(z)\right|^{\frac{1}{n_{k}}}$ and $h(z):=\psi^{*}(z)$ be a regularization of function $\psi$. Then it is known that the circular domain

$$
D:=\left\{z \in \mathbb{C}^{n}: h(z)<1\right\}
$$

is the domain of convergence of series (14). For this series we have the following multidimensional analogue of the Fabry theorem.

Theorem 6 ([4]). If for series (14)

$$
\varlimsup_{k \rightarrow \infty} \frac{k}{n_{k}}=\lim _{k \rightarrow \infty} \frac{k}{n_{k}}=0
$$

then the domain of convergence $D$ of series (14) coincides with the natural domain of existence $W_{f}$ of function $f(z)$.

Below, using the complex theory of pluripotential, we give more simple proof of this theorem, consider it in more general situations and give an analogue of the Polya theorem (the case when $\varliminf_{k \rightarrow \infty} \frac{k}{n_{k}}=0$ ). In the case when $\varliminf_{k \rightarrow \infty} \frac{k}{n_{k}}=0$, as it was noted above, series (14) can converge outside the domain $D$, i.e., $D$ is not the natural domain of existence of function $f$. Nevertheless, below we prove that $f$ is a single-valued function in $\mathbb{C}^{n}$, i.e. $W_{f} \subset \mathbb{C}^{n}$, and give a simple description of $W_{f}$ in terms of $\left.W_{f}\right|_{l}, \ell \in \mathbb{P}^{n-1}$.

Theorem 7. Let us assume that the following conditions for series (13) and for the family of complex lines $\mathcal{L} \subset \mathbb{P}^{n-1}$ hold:

1) $\mathcal{L}$ is not pluripolar;

2) $\varlimsup_{k \rightarrow \infty} \frac{k}{n_{k}}=0$;

3) for each complex line $\ell \in \mathcal{L}$ series (13) converges in the disk $l \cap B(0, r(l)), o<r \leqslant 1$.

Then the domain

$$
\Omega=\left\{z \in \mathbb{C}^{n}:|z| \exp V^{*}\left(\frac{z}{|z|}, E\right)<1\right\},
$$

is the domain of existence for the sum of series (13), where $E=\bigcup_{l \in \mathcal{L}}(l \cap B(0, r(l)))$.

As we noted earlier, if the radius of convergence $r(\ell) \leqslant R$ then the domain $\Omega$ in Theorem 7 has the form $\Omega=\left\{z \in \mathbb{C}^{n}: \frac{|z|}{R} \cdot \exp V^{*}\left(\frac{R z}{|z|}, E\right)<1\right\}$. We note that for a fixed complex line $\ell \ni 0$ the restriction of the series $\left.\sum_{s=0}^{\infty} \mathbb{Q}_{s}(z)\right]$ to this line determines a power series

$$
\sum_{s=0}^{\infty} \mathbb{Q}_{s}(\omega) w^{s}
$$

where $w \in \ell$ is the direction vector of the line $\ell$. If $\hat{R}(\omega)$ is the radius of convergence of series (15) then $r(\ell)=|\omega| \cdot \hat{R}(\omega)$. From this it follows that in the Siciak theorem the function

$$
h(z)=\frac{|z|}{R} \cdot \exp V^{*}\left(\frac{R z}{|z|}, E\right) .
$$

We also have the following multidimensional analogue of the Polya theorem for the lacunary series with respect to homogeneous polynomials with variable radius of convergence. 
Theorem 8. Let us assume that series (13) and the family $\mathcal{L} \subset \mathbb{P}^{n-1}$ of complex lines satisfy the following conditions

1) $\mathcal{L}$ is not pluripolar;

2) $\lim _{k \rightarrow \infty} \frac{k}{n_{k}}=0$;

3) for each straight line $\ell \in \mathcal{L}$ series (13) converges in a circle of positive radius $R(\ell)$.

Then series (13) defines a single-valued holomorphic function $f(z)$ in $\mathbb{C}^{n}$, that is, natural domain of existence $W_{f}$ of a function $f$ is schlicht, $W_{f} \subset \mathbb{C}^{n}$. Furthermore,

1) $0 \in W_{f}$;

2) $W_{f}$ coincides with the open core of the union $\bigcup_{\ell \ni 0} W_{f, \ell}$, i.e.

$$
W_{f}=\left(\bigcup_{\ell \in \mathbb{P}^{n-1}} W_{f, \ell}\right)^{0}
$$

3) for all complex lines $\ell \in \mathbb{P}^{n-1}$ except of some pluripolar set $P \subset \mathbb{P}^{n-1}$,

$$
\ell \cap W_{f}=W_{f, \ell}, \ell \notin P .
$$

Here we present a proof of Theorem 8. The proof of Theorem 7 follows from analogous reasoning and Theorem 1.

Proof of Theorem 8. From conditions of Theorem 8 it follows that series (13) satisfies conditions of Theorem 1. Then by Theorem 1 series (13) converges uniformly in some neighbourhood of zero, and its sum defines a function $f(z)$ that is holomorphic in this neighbourhood

$$
f(z):=\sum_{k=0}^{\infty} \mathbb{Q}_{n_{k}}(z) .
$$

Next, using a fractional-linear transformation (see Section 1), we map the region under consideration to the Hartogs domain. Then lines $\ell_{\ni} 0$ go in to straight lines parallel to the axis $z_{n}$ and a neighbourhood of zero in some domain $\mathbb{C}_{{ }_{z}}^{n-1} \times\left\{\left|z_{n}\right|<r\right\}, r>0$. Consequently, the series in homogeneous polynomials (16) becomes a Hartogs series

$$
g\left({ }^{\prime} z, z_{n}\right)=\sum_{k=0}^{\infty} c_{n_{k}}\left({ }^{\prime} z\right) z_{n}^{n_{k}}
$$

and this series satisfies conditions of Theorem 4:

Let series (17) satisfies the following conditions

1) series (17) converges uniformly inside the domain

$$
\mathbb{C}_{{ }_{z}}^{n-1} \times\left\{\left|z_{n}\right|<r\right\} \subset \mathbb{C}_{I^{n}}^{n-1} \times \mathbb{C}_{z_{n}}, r>0,
$$

2) $\varliminf_{k \rightarrow \infty} \frac{k}{n_{k}}=0$.

Then series (17) defines a single-valued holomorphic function $f(z, w)$ in the domain $W^{0}$, where $W^{0}$ is an open kernel of the set $W=\bigcup_{z \in D} W_{f}(z)$.

Using Theorem 4 and Proposition 2, we obtain the proof of Theorem 8. The theorem is proved. 


\section{References}

[1] E.Fabry, Sur les points singuliers d'une fonction donnee par son developpement de Taylor, Ann. ec. Norm. Sup. Paris, 13(1896), no. 3, 367-399.

[2] G.Faber, Uber Potenzreihen mit unendlich vielen verschiedenen koeffizienten, Sitzgsber. Bayr. Akad. Wiss., Mat.-nat. Abt., (1906), p. 581-583.

[3] G.Polya, Untersuchungen uber Luchen und Sungularitaten von potenzreihen, Math. Z., 29(1929), 549-640.

[4] J.Siciak, Some gap power series in multidimensional setting, Ann. Univ. Maria e CurieSklodowska Lublin-Polonia, 65(2011), no. 2, 179-190.

[5] A.Sadullaev, Plurissubharmonic functions, Current problems in mathematics. Fundamental directions, M., VINITI, 1985, Vol. 8, p. 65-113 (in Russian).

[6] A.Sadullaev, Pluripotential theory. Applications, Palmarium academik publishing, Saarbruchen Deutschland, 2012 (in Russian).

[7] T.Tuychiev, A.Mardanov, On domains of convergence of lacunary series with respect to homogeneous polynomials, Uzbek Mathematical Journal, 2014, no. 1, p. 108-118 (in Russian).

[9] A.Sadullaev, T.Tuychiev, On the extension of the Hartogs series admitting a holomorphic extension to parallel sections, Uzbek Mathematical Journal, (2009), no. 1, 148-157 (in Russian).

[8] M.Jarnicki, P.Pflug, Separately analytic functions, European Mathematical Society, 2011.

[10] A.Kolmogorov, S.Fomin, Elements of the theory of functions and functional analysis, Moscow, Nauka, 1976 (in Russian).

[11] T.Tuychiev, Continuation of functions along a fixed direction, Sibirsk. math. journal, 29(1988), no. 3, 142-147 (in Russian).

[12] T.Tuychiev, A.Mardanov, On multidimensional analogues of the Fabry and Polya theorems for lacunary Hartogs series, Uzbek Mathematical Journal, (2012), no. 3, 126-134 (in Russian).

[13] B.Shabat, Introduction to the complex analysis, Moscow, Nauka, 1985 (in Russian).

\section{Об областях сходимости многомерных лакунарных рядов}

Тахир Т. Туйчиев

Национальный Университет Узбекистана ВУЗ городок, Ташкент, 100174

Узбекистан

Данная работа посвящена многомерным аналогам теорем Фабри и Полиа о лакунарных рядах. В работе исследуются области сходимости лакунарных рядов Хартогса и рядов по однородным полиномам. Приводятся аналоги теорем Фабри и Полиа для таких рядов и описываются области сходимости этих рядов. Также развивается известный результат Й. Сичака об областях сходимости лакунарных рядов по однородным полиномам.

Ключевые слова: плюрисубгармоническая функиия, особая точка, пренебрежимо малые множества в $\mathbb{C}^{n}$, степенные ряды, лакунарные ряды Хартогса, ряды по однородным многочленам. 